Check for updates

Cite this: RSC Adv., 2019, 9, 9001

Received 6th February 2019

Accepted 5th March 2019

DOI: $10.1039 / c 9 r a 00981 g$

rsc.li/rsc-advances

\section{In situ Raman monitoring of dielectric-heating- enhanced freeze-drying under different electromagnetic wave frequencies}

\author{
S. Tsubaki, (D) *a T. Matsuzawa, ${ }^{\text {a }}$ S. Fujii, (iD ${ }^{\text {ab }}$ E. Suzuki, ${ }^{a}$ H. Kanamori, ${ }^{c}$ T. Hoshino, ${ }^{c}$ \\ S. Hosodac and Y. Wada ${ }^{\mathrm{C}}$
}

We studied the effect of dielectric heating on the enhancement of freeze-drying by electromagnetic waves (EMWs) under different frequencies: $2.45 \mathrm{GHz}$ microwaves (MWs), and 27 and $200 \mathrm{MHz}$ radio frequencies (RFs). The irradiation with RFs, particularly at $27 \mathrm{MHz}$, reduced the duration of freeze-drying by $67 \%$. We further analysed the water structure by in situ Raman spectroscopy during freeze-drying under EMWs. The phase transition from ice to water occurred soon after starting irradiation by MWs at $2.45 \mathrm{GHz}$, while the ice phase was almost maintained at an RF of $27 \mathrm{MHz}$.

\section{Introduction}

Dielectric heating by microwaves (MWs) and radio frequencies (RFs) enables rapid and direct heating of materials. Dielectric heating has been widely used for food processing, ${ }^{1}$ materials drying, ${ }^{2}$ processing, ${ }^{3}$ and various chemical reactions. ${ }^{4}$ Dielectric heating is capable of heating materials in a non-contact way. Thus, it can be used to heat materials with low heat conductivity (ca. vulcanisation of rubbers) $)^{5}$ or under vacuum conditions ( $c a$. MW-assisted vacuum drying, ${ }^{6}$ and $\mathrm{MW}$-assisted extraction of essential oils $\left.{ }^{7}\right)$. Freeze-drying is an important process in the food industry as well as pharmaceutical production. The enhancement of freeze-drying is strongly desired because freeze-drying is generally a time-consuming and costly process. Dielectric heating can enhance the rate of freeze-drying by directly distributing energy to frozen samples under vacuum, so MWs have previously been used to enhance the freeze-drying of foods. ${ }^{8-10}$

Dielectric heating occurs via the interaction of applied electromagnetic waves (EMWs) and irradiated materials by two mechanisms: dielectric and conduction losses. ${ }^{11}$ Dielectric loss originates from the orientation and relaxation of dipoles, while conduction loss is attributed to the displacement of charges. Therefore, the complex dielectric constants of materials, which indicate the degree of EMWs absorbed by materials, are important parameters for the effective heating of materials by

${ }^{a}$ School of Materials and Chemical Technology, Tokyo Institute of Technology, Ookayama 2-12-1 E4-3, Meguro, Tokyo, 152-8550, Japan. E-mail: shuntaro. tsubaki@gmail.com; tsubaki.s.aa@m.titech.ac.jp

${ }^{b}$ Department of Information and Communication Systems Engineering, Okinawa National College of Technology, 905 Henoko, Nago-shi, Okinawa 905-2192, Japan

cJapan Aerospace Exploration Agency, 3-1-1 Yoshinodai, Chuo-ku, Sagamihara, Kanagawa, 252-5210, Japan
EMWs. The dielectric parameters vary depending on the materials, temperature, and applied frequency of the EMWs. For example, the complex dielectric constant of water significantly changes depending on its phase. Liquid water at $273 \mathrm{~K}$ undergoes maximum absorption of EMWs at $10 \mathrm{GHz}$, while ice water at the same temperature reaches the maximum absorption at $3 \mathrm{kHz} \cdot{ }^{12-15}$ The appropriate frequency of the EMWs is important for obtaining efficient energy transfer.

In this study, we tested different frequencies of EMWs in a frequency range between RFs and MWs to enhance the freezedrying of model aqueous solutions of pure water and $\mathrm{NaCl}$. First, we determined the complex dielectric constants of the samples in a wide frequency range using the coaxial probe method to evaluate their frequency- and temperaturedependent dielectric properties. We then built a MW (2.45 $\mathrm{GHz}$ ) and $\mathrm{RF}$ (27 and $200 \mathrm{MHz}$ ) irradiation systems to apply different frequencies of EMWs to the freeze-drying of the model aqueous solutions. We also analysed the water structure during freeze-drying under EMWs by in situ Raman spectroscopy to understand the mechanism of freeze-drying enhancement.

\section{Experimental}

Measurement of the temperature-dependent dielectric properties of water and the aqueous $\mathrm{NaCl}$ solution

The complex dielectric constants of water, aqueous $\mathrm{NaCl}$ solutions ( 0.05 and $0.1 \mathrm{M}$ ) were measured by a coaxial probe method using a KEYCOM Co. probe-type kit (open mode) and Rhode \& Schwartz ZND network analyser in the $200 \mathrm{MHz}-8.5 \mathrm{GHz}$ frequency range. The temperature of the samples was controlled between -20 and $30{ }^{\circ} \mathrm{C}$ in an aluminium bead bath connected to a chiller. Dielectric spectra were obtained when the temperature became constant at the desired temperature. 
MW and RF heating systems for freeze-drying of water and aqueous $\mathrm{NaCl}$ solution

The MW and RF heating systems are illustrated in Fig. 1. The MW heating was conducted using a single mode $\left(\mathrm{TM}_{110}\right)$ cavity resonator equipped with a semiconductor MW generator (2.45 GHz, Chronix Co.). The RF heating at $200 \mathrm{MHz}$ was conducted using a parallel plate applicator (200 $\mathrm{MHz}$, Fuji Electronic Ind.) equipped with a signal generator (8648C Keysight Technologies) and a GaN RF amplifier (ZHL-100W-GAN+, Mini-Circuits, frequency range $20-500 \mathrm{MHz}$, max. output $100 \mathrm{~W}$ ) as indicated in our previous report. ${ }^{16}$ The RF heating at $27 \mathrm{MHz}$ was conducted with a parallel plate applicator $(27 \mathrm{MHz}$, Fuji Electronic Ind.) equipped with a $27 \mathrm{MHz}$ RF generator (Fuji Electronic Ind.). The applied power was monitored using power monitors connected to each system.

The freeze-drying samples (water and aqueous $\mathrm{NaCl}$ solutions; $3 \mathrm{~mL}$ ) were added to a flat-bottom glass test tube ( $\phi 18$ $\mathrm{mm}$ ) equipped with a two-way valve and pre-frozen in a freezer. The temperature of the trap in the freeze-dryer (FDU-1200, EYELA) and suction rate of the pump (GOD-051XF, EYELA) were set to $-46{ }^{\circ} \mathrm{C}$ and $50 \mathrm{~L} \mathrm{~min}^{-1}$, respectively. The test tubes were connected to the freeze-dryer by a vacuum hose and placed inside the MW and RF applicators as described in Fig. 1. MWs or RFs were continuously applied to the test tube at $10 \mathrm{~W}$. The control experiment was conducted using the same freeze-dryer

\section{(A) $2.45 \mathrm{GHz}$ MW system}

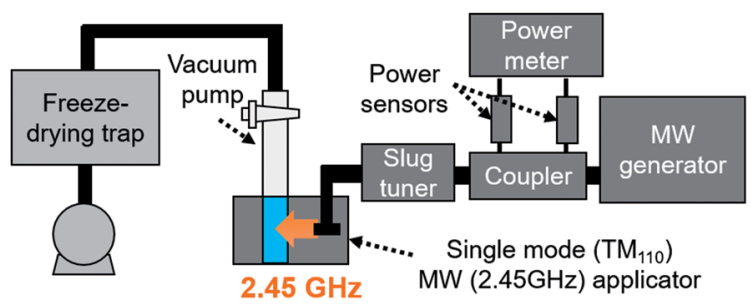

(B) 200 MHz RF system

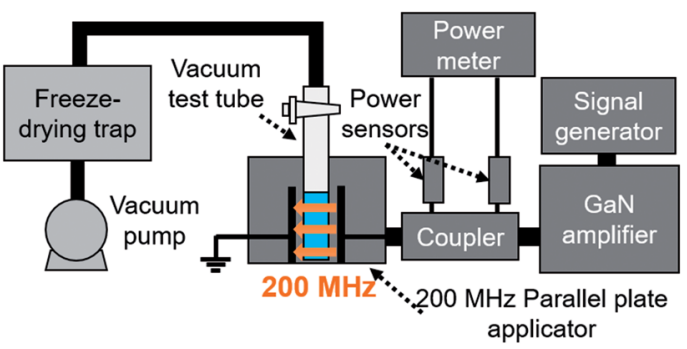

(C) 27 MHz RF system

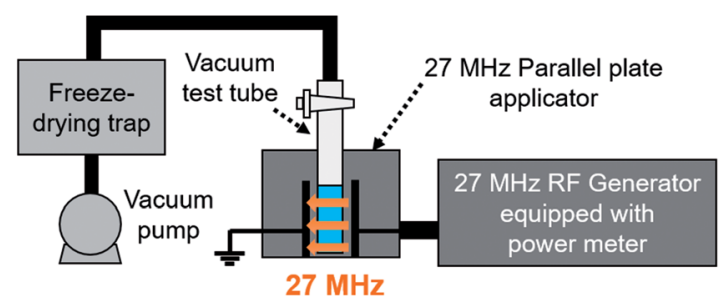

Fig. 1 Freeze-drying systems under $M W$ and RF irradiation: (A) 2.45 $\mathrm{GHz} \mathrm{MW}$, (B) $200 \mathrm{MHz} R \mathrm{R}$, and (C) $27 \mathrm{MHz}$ RF. without MW or RF irradiation. The weight of the whole test tube was measured every 5-20 min. The irradiation of the MWs or RFs were stopped after the weight of the test tube became constant.

In situ Raman monitoring of water structure during freezedrying of water under RF (27 MHz) and MW (2.45 GHz) irradiation

The structure of water during freeze-drying under MW and RF irradiation was monitored using Raman spectroscopy. A typical in situ Raman monitoring system under RF (27 MHz) irradiation is illustrated in Fig. 2. A Raman head (MobileRAM/H/TKT, Lambda Vision) equipped with an objective lens (Mitutoyo $\mathbf{M}$ Plan Apo $10 \times$ ) was placed below the MW and RF applicators. The $532 \mathrm{~nm}$ laser (MSL-FN-532-100 m, Changchun New Industries Optoelectronics Technology) was used as a light source. The Raman spectra were obtained using a Horiba iHR320 spectrometer and Horiba Syncerity CCD detector. The highresolution Raman spectra were obtained every 10 min with $5 \mathrm{~s}$ of laser irradiation time and five scans. In the case of transient Raman monitoring of the ice and water structure, the Raman spectra were continuously obtained with $1 \mathrm{~s}$ of laser irradiation time with 1 scan for each spectrum.

\section{Results and discussion}

Temperature-dependent dielectric properties of water and the aqueous $\mathrm{NaCl}$ solution

The dielectric properties of water and an aqueous salt solution were first measured for the purpose of analysing their dependencies on phase, temperature, and frequency of EMW. Fig. 3 illustrates the temperature-dependent dielectric properties of water and the aqueous $\mathrm{NaCl}$ solution. The real part of the complex dielectric constant (relative permeability, $\varepsilon^{\prime}$ ) indicates the degree of EMW energy stored in a material, while the

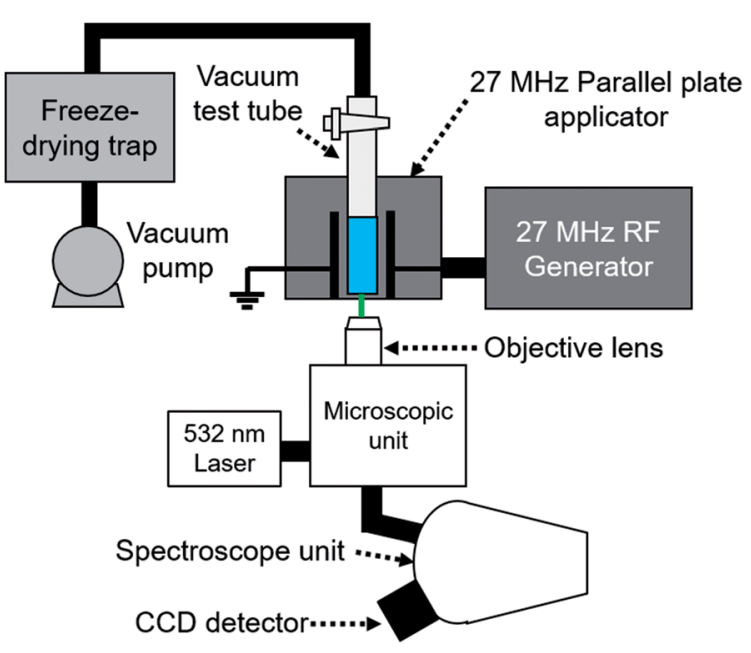

Fig. 2 Schematic illustration of in situ Raman spectroscopy during freeze-drying of ice under RF irradiation at $27 \mathrm{MHz}$. The in situ Raman spectroscopy under MW irradiation at $2.45 \mathrm{GHz}$ was conducted using the same set-up. 
(A)

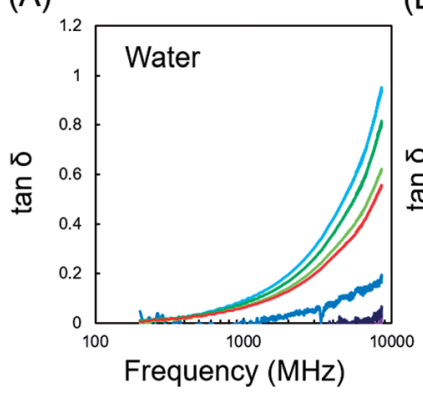

(B)
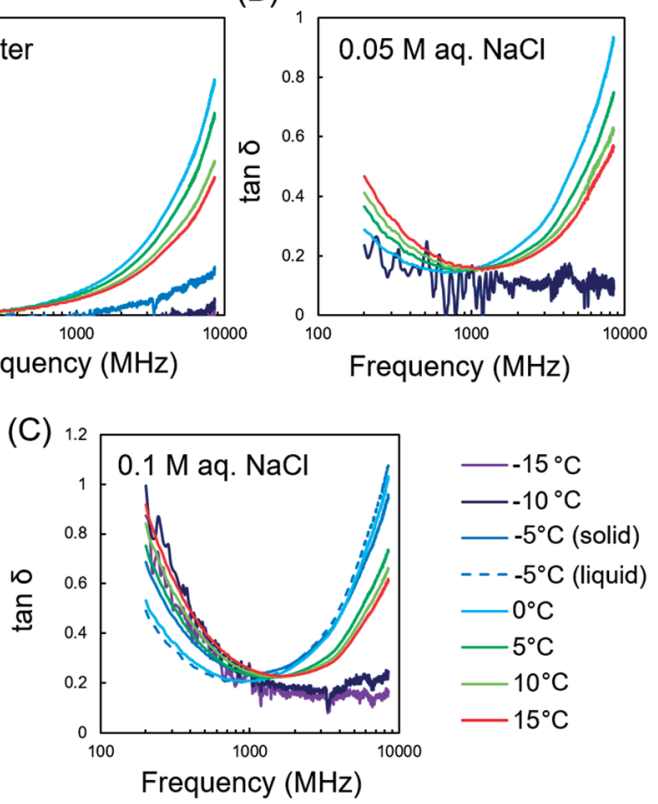

Fig. 3 Temperature-dependent dielectric properties of the (A) water, (B) $0.05 \mathrm{M}$ aqueous $\mathrm{NaCl}$ solutions, and (C) $0.1 \mathrm{M}$ aqueous $\mathrm{NaCl}$ solution, from -15 to $15^{\circ} \mathrm{C}$.

imaginary part (the sum of dielectric and conduction loss, $\varepsilon^{\prime \prime}$ ) indicates the degree of loss of the EMW energy dissipated in the materials. ${ }^{11}$ The degree of EMWs absorbed in the materials are described by the dielectric loss tangent $(\tan \delta)$, which is the ratio of $\varepsilon^{\prime \prime} \mid \varepsilon^{\prime}$. The dielectric property of water changes with phase transitions. ${ }^{13}$ The $\tan \delta$ values of ice were very small. However, these drastically increased after the phase transition to liquid water above $0{ }^{\circ} \mathrm{C}$, especially for higher frequencies $(>1 \mathrm{GHz})$ due to the dipole rotation of water molecules (Fig. 3A). The further increase in temperature led to a gradual decrease in the $\tan \delta$ values which attributes to the increased mobility of water molecules. ${ }^{17}$

The aqueous $\mathrm{NaCl}$ solution exhibited an elevated $\tan \delta$ at lower frequencies $(<1 \mathrm{GHz}$, Fig. 1B), which was attributed to conduction loss by ions. ${ }^{11}$ The increased concentration of $\mathrm{NaCl}$ provided a more prominent increase in the $\tan \delta$ values (Fig. 1C). In contrast, the $\tan \delta$ values of the aqueous $\mathrm{NaCl}$ solution increased with increasing temperature since the ionic conduction became more prominent at elevated temperatures, similar to that previously observed for electrolyte polymers in water. ${ }^{18}$ The high $\tan \delta$ value was even observed in the solid phase of $0.1 \mathrm{M} \mathrm{NaCl}$, which is likely due to the non-freezing bound water generated by the addition of electrolytes in water. This is originated from hydrated ions that change the water structure depending on the ion species. ${ }^{19}$

According to the temperature dependencies of the $\tan \delta$ values of ice and water, the appropriate EMW frequency should be used for freeze-drying to prevent overheating of the irradiated materials. The use of RFs is preferred, thus avoiding overheating of liquid water because its dielectric loss is very small in the RF range. MWs at $2.45 \mathrm{GHz}$ can cause unexpected heating when liquid water is generated during freeze-drying because liquid water exhibits a higher dielectric loss than ice at $2.45 \mathrm{GHz}$. However, when concentrated electrolytes are in water, RF irradiation can also cause heating because the conduction loss becomes prominent in the RF range even in a solid phase.

\section{MW and RF-assisted freeze-drying of water and the aqueous NaCl solution}

A freeze-drying system equipped with MW and RF heating devices was built to test the effect of different EMW frequencies on freeze-drying in every octave from $27 \mathrm{MHz}$ to $2.45 \mathrm{GHz}$ (Fig. 1). Fig. 4 shows a comparison of the effect of different EMW frequencies on the freeze-drying of water. The application of EMWs greatly reduced the duration of freeze-drying by $67 \%$ (27 MHz) to $75 \%(2.45 \mathrm{GHz})$. Therefore, the EMWs directly provided energy for the sublimation of ice, which is much faster than heat convection through the wall of the glass tube. The MWs provided a slightly higher freeze-drying rate compared with the RFs at the same applied power. However, water generation was promptly observed after starting MW irradiation. At the same time, the pressure in the freeze-dryer increased to $\sim 80-90 \mathrm{~Pa}$, which further induced ice melting. Ice melting should be avoided, especially when freeze-drying pharmaceuticals that are very sensitive to heat. In contrast, ice phase was maintained during freeze-drying under RF irradiation at $27 \mathrm{MHz}$.

The contribution of adding $\mathrm{NaCl}$ was further studied as some foods contain salts. Fig. 5 shows the weight change of the 0.05 and $0.1 \mathrm{M}$ aqueous $\mathrm{NaCl}$ solutions, with and without 27 $\mathrm{MHz}$ RF irradiation. The RF case resulted in a reduced duration of freeze-drying by $71 \%$. However, ice melting could not be prevented. This is attributed to the elevated $\tan \delta$ of the aqueous $\mathrm{NaCl}$ solution in the RF range (Fig. 3B and C). Tanaka et al. previously reported the heating of ice water by microwaves due to the interaction of ions in ice with EMWs, using a molecular dynamics simulation. ${ }^{20}$ The frozen-state of irradiated material

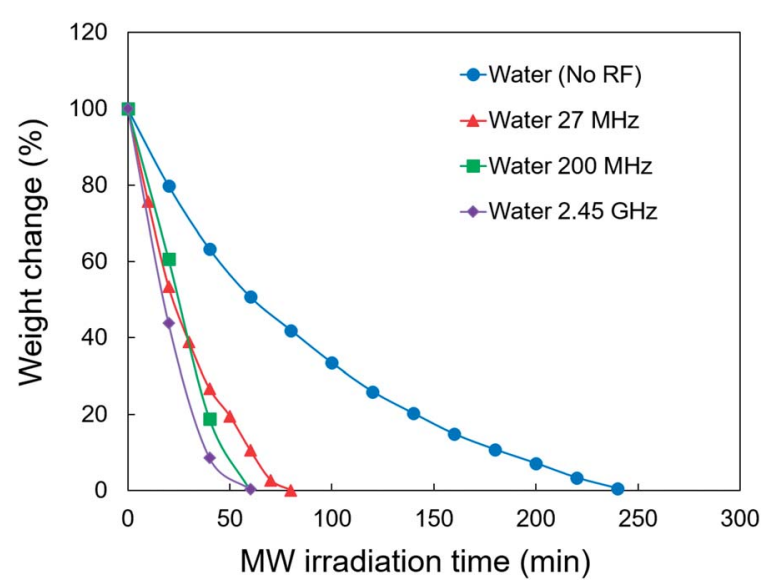

Fig. 4 Weight changes of the freeze-drying of water with and without $\mathrm{RF}$ and $\mathrm{MW}$ irradiation. 


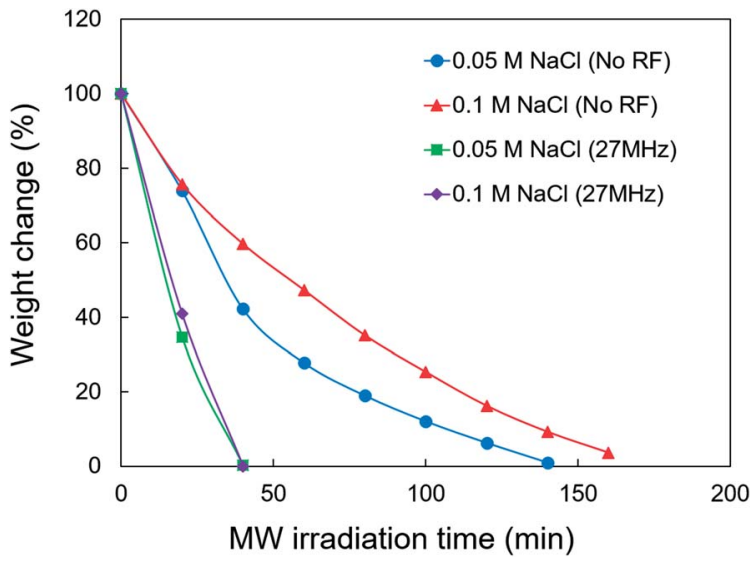

Fig. 5 Weight changes of the freeze dried aqueous $\mathrm{NaCl}$ solution with and without RF irradiation (27 MHz)

should be carefully controlled when freeze-drying samples containing large amounts of electrolytes.

\section{In situ Raman monitoring of the water structure during freeze-drying under RF and MW irradiation at $27 \mathrm{MHz}$ and $2.45 \mathrm{GHz}$, respectively}

In situ Raman monitoring of the water structure during freezedrying was conducted to verify the water phase under EMW irradiation. Raman spectroscopy has been used to characterise water structure in a non-contact way. Thus, it is a powerful tool for determining the water phase during food processing. ${ }^{21-25}$ The $\mathrm{OH}$-stretching band in the range $2900-3700 \mathrm{~cm}^{-1}$ is a good indicator of the water structure because it is sensitive to the hydrogen bond networks in water.

Fig. 6 shows the Raman spectra during freeze-drying of pure water for wavenumbers between 2500 and $4000 \mathrm{~cm}^{-1}$. The sharp peak at $3114 \mathrm{~cm}^{-1}$ corresponds to the symmetric O-H stretching band, which is indicative of a fully hydrogen bonded water molecule. The shoulder peak near $3410 \mathrm{~cm}^{-1}$ indicates an asymmetric $\mathrm{O}-\mathrm{H}$ stretching band, attributed to water molecules with hydrogen bonds that are partly or totally broken. ${ }^{15,16}$ Therefore, the liquid water exhibited a broad peak for 2900$3700 \mathrm{~cm}^{-1}$, while the ice water exhibited a sharp peak at $3114 \mathrm{~cm}^{-1}$.

The ice phase was kept constant during freeze-drying for the case without EMW irradiation (Fig. 6A). The Raman spectra under $27 \mathrm{MHz}$ RF irradiation remained almost the same as that of ice (Fig. 6B), while that under $2.45 \mathrm{GHz}$ irradiation exhibited a significant phase transition from ice to water within $10 \mathrm{~min}$ (Fig. 6C). The Raman spectra for the $2.45 \mathrm{GHz}$ case was further obtained in a shorter period as indicated in Fig. 6D. A phase transition drastically occurred between 135 and $172 \mathrm{~s}$ after starting MW irradiation, indicating that the phase transition of ice occurred at the beginning of freeze-drying.

Although the $\mathrm{O}-\mathrm{H}$ stretching band is broad and convoluted in several components, the ratio of the symmetric and asymmetric stretching $\mathrm{O}-\mathrm{H}$ bands provides information on the phase transitions of water. ${ }^{23}$ Fig. 7 shows the time-dependent
(A)

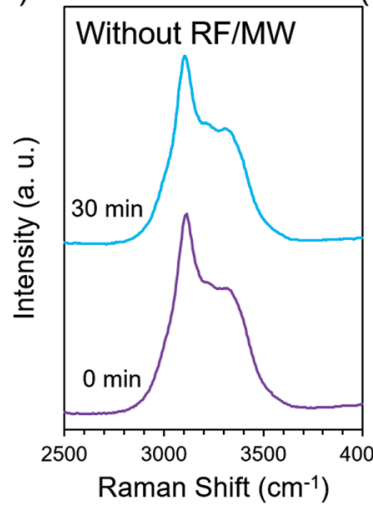

(B)

(C)

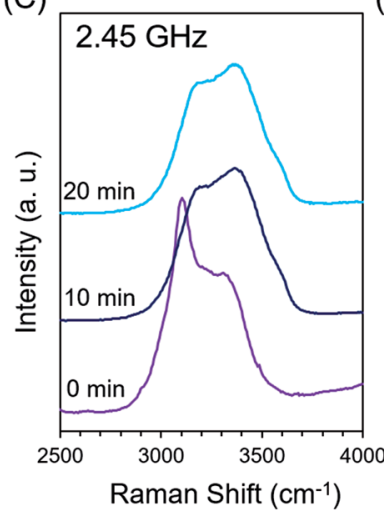

(D)

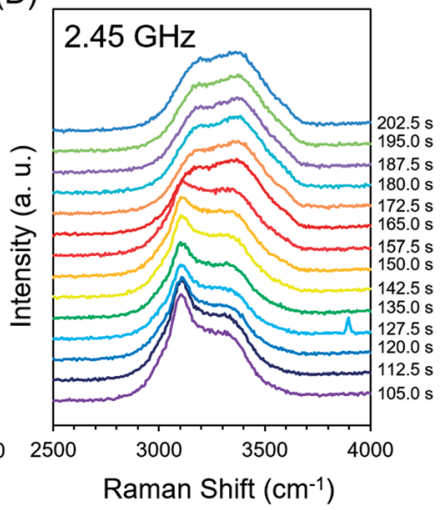

Fig. 6 In situ Raman monitoring of the water structure during the freeze-drying of water, with and without EMW irradiation: (A) without EMW (0-30 min), (B) $27 \mathrm{MHz}(0-60 \mathrm{~min}),(\mathrm{C}) 2.45 \mathrm{GHz}(0-20 \mathrm{~min})$, and (D) $2.45 \mathrm{GHz}(105.0-202.5 \mathrm{~s})$.

ratios of the intensities of the peaks at 3114 and $3410 \mathrm{~cm}^{-1}$ $[I(3410) / I(3114)]$ indicating the degree of water freezing, both with and without EMW irradiation. A constant value was

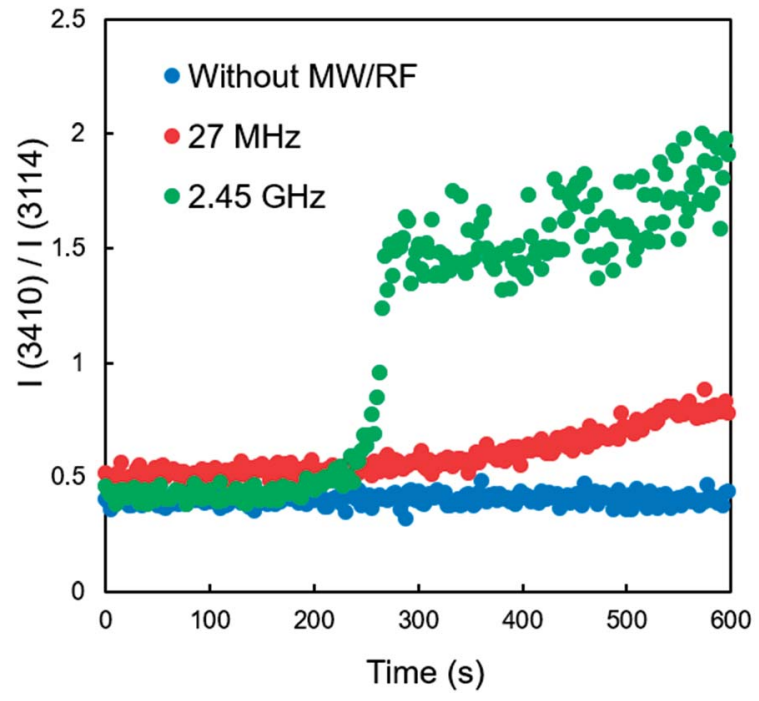

Fig. 7 Changes in the ratios of the Raman peak intensity /(3410)/ I(3114) during the freeze-drying of water, with and without RF and MW irradiation. 
obtained during freeze-drying without EMW irradiation. The 27 MHz RF irradiation produced a slight increase in the value of $I(3410) / I(3114)$, indicating that a slight structural change occurs in ice during RF irradiation, even when the sample looks like the remaining ice phase. In contrast, the $2.45 \mathrm{GHz} \mathrm{MW}$ irradiation produced an instantaneous increase within a short period, which was attributed to the significant phase transition from ice to water. The generated water absorbed MWs to produce more heat, which further induced the melting of the ice because the dielectric loss of water was much higher than ice for the $2.45 \mathrm{GHz}$ irradiation. Therefore, RF irradiation is preferred for the enhancement of freeze-drying of heat-sensitive materials such as biological samples because heat generation should be avoided.

\section{Conclusions}

We studied the effect of EMW irradiation using different frequencies (2.45 GHz MWs, and 27 and $200 \mathrm{MHz}$ RFs) for the purpose of enhancing the freeze-drying rate. Measurements of the complex dielectric constant indicated a prominent increase in the dielectric loss during the phase transition from ice to water, particularly in the MW range above $1 \mathrm{GHz}$. The direct heating by EMW irradiation enhanced the sublimation of water and reduced the duration of ice freeze-drying by $67-75 \%$. The freeze-drying rate using $27 \mathrm{MHz} \mathrm{RF}$ irradiation was slightly slower than those of other frequencies. However, water generation was avoided. The water structure during freeze-drying with and without EMW irradiation was further analysed using in situ Raman spectroscopy. The RF irradiation modified the water structure slightly. However, a frozen appearance remained. In contrast, the phase transition from ice to water occurred soon after exposure to MW irradiation. The MWs induced water generation, which facilitated further increases in the dielectric loss, increasing the MW susceptibility. RF irradiation, especially at $27 \mathrm{MHz}$, is therefore preferred for enhancing the freeze-drying rate, while maintaining the frozen phase of the material.

\section{Conflicts of interest}

There are no conflicts to declare.

\section{Acknowledgements}

This work was supported in part by the grant "Open Innovation Hub for Expanding Humanosphere and Domain of Human Activity through Solar System Frontier Development" from the Japan Aerospace Exploration Agency (JAXA) Space Exploration Innovation Hub Center, and Japan Science and Technology Agency (JST); Grant-in-Aid for Scientific Research (A) 25249113; Grant-in-Aid for Scientific Research (S) 17H06156; and JSPS Grant-in-Aid for Young Scientists (A) $17 \mathrm{H} 05049$.

\section{Notes and references}

1 S. Chandrasekaran, S. Ramanatha and T. Basak, Food Res. Int., 2013, 52, 243-261.

2 M. Zhang, J. Tang, A. S. Mujumdar and S. Wang, Trends Food Sci. Technol., 2006, 17, 524-534.

3 H. J. Kitchen, S. R. Vallance, J. L. Kennedy, N. Tapia-Ruiz, L. Carassiti, A. Harrison, A. G. Whittaker, T. D. Drysdale, S. W. Kingman and D. H. Gregory, Chem. Rev., 2014, 114, 1170-1206.

4 S. Horikoshi and N. Serpone, Microwaves in Catalysis: Methodology and Applications, Wiley-VCH, 2015.

5 M. Akiba and A. S. Hashim, Prog. Polym. Sci., 1997, 22, 475521.

6 R. H. Walters, B. Bhatnagar, S. Tchessalov, K.-I. Izutsu, K. Tsumoto and S. Ohtake, J. Pharm. Sci., 2014, 103, 26732695.

7 N. Flórez, E. Conde and H. Domínguez, J. Chem. Technol. Biotechnol., 2015, 90, 590-607.

8 X. Duan, M. Zhang, A. S. Mujumdar and S. Wang, J. Food Eng., 2010, 96, 491-497.

9 X. Duan, M. Zhang, S. Mujumdar and R. Wang, Drying Technol., 2010, 28, 444-453.

10 X. Duan, M. Zhang and A. S. Mujumdar, Drying Technol., 2007, 25, 1725-1731.

11 C. Gabriel, S. Gabriel, E. Grant, H. Grant, B. S. J. Halstead, D. Michael and P. Mingos, Chem. Soc. Rev., 1998, 27, 213223.

12 A. von Hippel, IEEE Trans. Electr. Insul., 1988, 23, 801-816.

13 J. L. Aragones, L. G. MacDowell and C. Vega, J. Phys. Chem. A, 2011, 15, 5745-5758.

14 V. G. Artemov and A. A. Volkov, Ferroelectrics, 2014, 466(1), 158-165.

15 A. A. Volkov, V. G. Artemov and A. V. Pronin, Dokl. Phys., 2014, 59, 111-114.

16 S. Tsubaki, S. Hayakawa, T. Ueda, S. Fujii, E. Suzuki, J. Zhang, A. Bond and Y. Wada, Chem. Commun., 2019, 55, 1032-1035.

17 K. Okada, M. Yao, Y. Hiejima, H. Kohno and Y. Kajihara, J. Chem. Phys., 1999, 110, 3026.

18 S. Tsubaki, M. Hiraoka, S. Hadano, K. Okamura, T. Ueda, H. Nishimura, K. Kashimura and T. Mitani, Carbohydr. Polym., 2015, 115, 78-87.

19 H. J. Bakker, Chem. Rev., 2008, 108, 1456-1473.

20 M. Tanaka and M. Sato, J. Chem. Phys., 2008, 126, 034509.

21 G. E. Walrafen, J. Chem. Phys., 1964, 40, 3249-3256.

22 B. Minceva-Sukarova, W. F. Sherman and G. R. Wilkinson, J. Phys. C: Solid State Phys., 1984, 17, 5833-5850.

23 I. Đuričković, R. Claverie, P. Bourson, M. Marchetti, J.-M. Chassot and M. D. Fontana, J. Raman Spectrosc., 2011, 42, 1408-1412.

24 X. Xu, Z.-Z. He and J. Jiu, J. Raman Spectrosc., 2013, 44, 10451048.

25 Q. Hu, S. Ouyang, J. Li and Z. Cao, J. Raman Spectrosc., 2017, 48, 610-617. 